\title{
Correction to: Systemic inflammatory responses after orthopedic surgery in patients with rheumatoid arthritis treated with tofacitinib
}

\author{
Akihiro Uchio ${ }^{1,2} \cdot$ Takumi Matsumoto $^{1}$ (1) - Yuji Maenohara ${ }^{1} \cdot$ Yasunori Omata ${ }^{1} \cdot$ Hiroshi Takahashi $^{3}$. \\ Mitsuyasu Iwasawa ${ }^{2} \cdot$ Takuo Juji $^{3} \cdot$ Ichiro Nakamura $^{3} \cdot$ Sakae Tanaka ${ }^{1} \mathbb{C}$
}

Published online: 1 November 2021

(c) International League of Associations for Rheumatology (ILAR) 2021

\section{Correction to: Clinical Rheumatology \\ https://doi.org/10.1007/s10067-021-05914-1}

In the original published version of this article, the First author's name was presented incorrectly. The name should have been presented as "Akihiro Uchio" instead of "Uchio Akihiro". The name is now presented correctly above.

The original article has been corrected.

Publisher's note Springer Nature remains neutral with regard to jurisdictional claims in published maps and institutional affiliations.

The original article can be found online at https://doi.org/10.1007/ s10067-021-05914-1.

Takumi Matsumoto

matumot-tky@umin.ac.jp

1 Department of Orthopaedic Surgery, Faculty of Medicine,

The University of Tokyo, 7-3-1, Hongo, Bunkyo-ku,

Tokyo 113-8655, Japan

2 Department of Orthopaedic Surgery, National Hospital Organization, Sagamihara Hospital, 18-1 Sakuradai, Minami-ku, Sagamihara City, Kanagawa 252-0314, Japan

3 Department of Rheumatology, JCHO Yugawara Hospital, 2-21-6 Chuo, Yugawara, Ashigara-shimo, Kanagawa 259-0396, Japan 